\title{
UN MODELO MATEMÁTICO SOBRE LA TEORÍA DE JUEGOS
}

ADEI ARCE CARRASCO

Tres economistas que emplearon el estudio de los juegos para analizar las finanzas, ganaron el Premio NOBEL de Economia de 1994.

Los norteamericanos: John Harsangi y John Nash, asi como el alemán Reinhard Selten. refinaron los fundamentos de la Teoria de Juegos, descubierta hace 50 años, para realizar predicciones a través de estrategias basadas en factores desconocidos.

La teoria moderna de juegos fue desarrollada en la década del 40 por John Von Neumann y Oscar Morgenstem, para dar un modelo matemático a la economia. Además, se desarrolló con el propósito de analizar situaciones competitivas que involucrasen intereses conflictivos durante la segunda guerra mundial. Las ideas principales de esta Teoria se extrajeron de juegos bien conocidos como el ajedrez, el solitanio, el póker, etc, Hoy se puede explicar al análisis de cualquier comportamiento competitivo; en la economia, en la guerra, en los negocios, en el comportamiento social, etc.

El juego se caracteriza por sus reglas; en algunos casos el juego puede ser tan complicado debido a que las reglas son $\tan$ complejas, por ejemplo las diferentes acciones que se dan en un sistema social humano, o de un sistema ecológico en que intervienen muchos contendores.

Cuando ya se conocen las reglas, el problema consiste en determinar la manera en que los jugadores deben escoger sus movimientos; es decir, los jugadores deben seleccionar mejores opciones analizando las reglas del juego. Los juegos se clasifican respecto a su solución en:

\section{Por el número de personas, o por el nú- mero de intereses.}

Al conjunto de personas que tienen un determinado objetivo se le considera como un solo jugador. Hasta ahora la Teoría se ha desarrollado para el juego entre dos intereses. entre dos objetivos. El análisis de juegos que involucran más de dos personas es usualmente muy dificil.

\section{Por la naturaleza del juego.}

La pregunta inmediata surge: ¿qué pasa al final del juego? Si la suma de los pagos que se efectua a los dos jugadores de un juego es cero. considerando las ganancias como positivas y las pérdidas como negativas, entonces se dice que el juego es de suma cero.

Casi todos los juegos de Salón son juegos de suma cero los juegos de suma no cero necesita de un análisis un poco más refinado.

\section{Por las estrategias empleadas.}

Una estrategia es un plan completo que utiliza cada jugador, sin ninguna habilidad marcada; esto es, emplear sus estrategias sin "trampas".

Los juegos de garito tales como los dados, la ruleta, no son juegos de estrategia. Una persona que juega uno de éstos, está "jugando a la suerte", no contra un oponente conciente o racional.

La idea de estrategia se debe definir matemáticamente, para ello es necesario introducir conceptos preliminares sobre la teoria de probabilidades, algebra lineal, etc. Existen dos tipos de estrategia fundamentaimente: 
estrategias puras y estrategias mixtas según como se caracterizan los juegos.

\section{ESTRATEGIAS}

La Teoria de Juegos asume un tipo particular de situación que involucra la maximización de valor esperado de una decisión hecha bajo la incertidumbre $y$, como sabemos, la probabilidad es una medida numérica de la incertidumbre.

Cada jugador desea maximizar sus beneficios minimos esperados, o equivalentemente, desea minimizar sus perdidas máximas esperadas. Esta idea se basa en un criterio llamado "maximin" y "minimax" respectivamente. Esto constituye la base para la Teoria de Juegos y que hoy en dia se ha extendido por la investigación a varias áreas de aplicación.

\section{DEFINICIÓN}

1. Sea $A=\left(a_{1}\right)$ una matriz de orden $m \neq n$. Considere un juego determinado por Aentre dos competidores $\boldsymbol{X}$ y $\boldsymbol{Y}$, de acuerdo a las siguientes reglas:

(a) En cada movimiento del juego, $X$ escoge una de las $m$ filas, y $Y$ escoge una de las $\boldsymbol{n}$ columnas de A. Estas elecciones se realizan simultáneamente y ninguno de los jugadores sabe de antemano la elección del otro competidor:

(b) Si $X$ escoge la fila " $i$ ", y $Y$ escoge la columna " $j "$, entonces $X$ gana a $Y$ la cantidad $\boldsymbol{a}_{\boldsymbol{f}}$, si $\boldsymbol{a}_{\mathrm{f}}$ es positivo; $\boldsymbol{i}$ pierde, si $\boldsymbol{a}_{\boldsymbol{i}}$ es negativo.

Este juego como tal se llama juego de matriz, i la matriz A recibe el nombre de matriz de pagos to también matriz de juego).

2. Los dos jugadores deben analizar sus posibles movimientos y decidir en qué filas o columnas juegan sus movimientos sucesivos. Si $\boldsymbol{X}$ decide jugar en una misma fila y $Y$ en una misma columna en cada movimiento del juego; se dice que estàn usando una estrategia pura. Por el contrario. si $\boldsymbol{X}$ decide jugar en otra fila y $\boldsymbol{Y}$ en otra columna, en movimientos distintos del juego. diremos que están usando una estrategia mixta.

3. Una estrategia para el jugador $X$ es un vector de probabilidad $\boldsymbol{p}=\left(\boldsymbol{p}_{r}, \boldsymbol{p}_{z}, \ldots, \boldsymbol{p}_{m}\right)$ donde $\boldsymbol{p}$, es la probabilidad de que $X$ juegue escogiendo la fila "i", $0 \leq i \leq m$. Una estrategia para $Y$ es un vector de probabilidad $\boldsymbol{q}=\left(\boldsymbol{q}_{1}, \boldsymbol{q}_{2}, \ldots, \boldsymbol{q}_{n}\right)$, donde $\boldsymbol{q}_{j}$ es la probabilidad de queAY juegue escogiendo la columna " $j$ ", $\sigma \leq j \leq m$.

4. Si una matriz de pagos contiene una entrada $a_{j}$ que es simultáneamente un maximo de filas minimas (maximin) y un minimo de columnas máximas (minimax): esta entrada se llama un punto de silla del juego, y el juego se dice que es estrictamente determinado. De acuerdo al criterio de la Teoria de Juegos, el jugador $\boldsymbol{X}$ debe jugar escogiendo la fila " $i$ ". $y$ el jugador $Y$, escogiendo la columna " $j$ ". Con estas jugadas ambos competidores están empleando sus estrategias óptimas. Si existe el punto de silla, el juego está resuelto. Si no existe el punto de silla, se dice que el juego es no-estrictamente determinado, y prosigue el juego siguiendo un análisis posterior.

Las estrategias disponibles a cada jugador se puede enumerar, y los correspondientes pagos se pueden expresar en números (puede ser en nuevos soles, aunque no necesariamente debe ser monetario). Al finalizar un juego, el valor de un juego es el pago promedio esperado que recibe un jugador, supuesto que han utilizado sus estrategias óptimas en forma 
consciente. Cuando el valor es cero, se dice que el juego es justo. En un juego justo ningún jugador tiene ventaja. En resumen, cada jugador desea maximizar sus ganancias minimas o minimizar sus pérdidas máximas.

En general, dada la matriz de pagos $A=(a)$ de $m \times n$; mediante el cuadro siguiente se determina un punto de silla. Por convención, las estrategias para el jugador $\boldsymbol{X}$ están indicadas a lo largo de una columna situada en la parte de la izquierda de la matriz de pagos, y las estrategias para el jugador $Y$ están indicadas a lo largo de una fila situada en la parte superior.

\section{JUGADOR $Y$}

(1) (2) .... (n)

JUGADOR $\mathrm{x}$

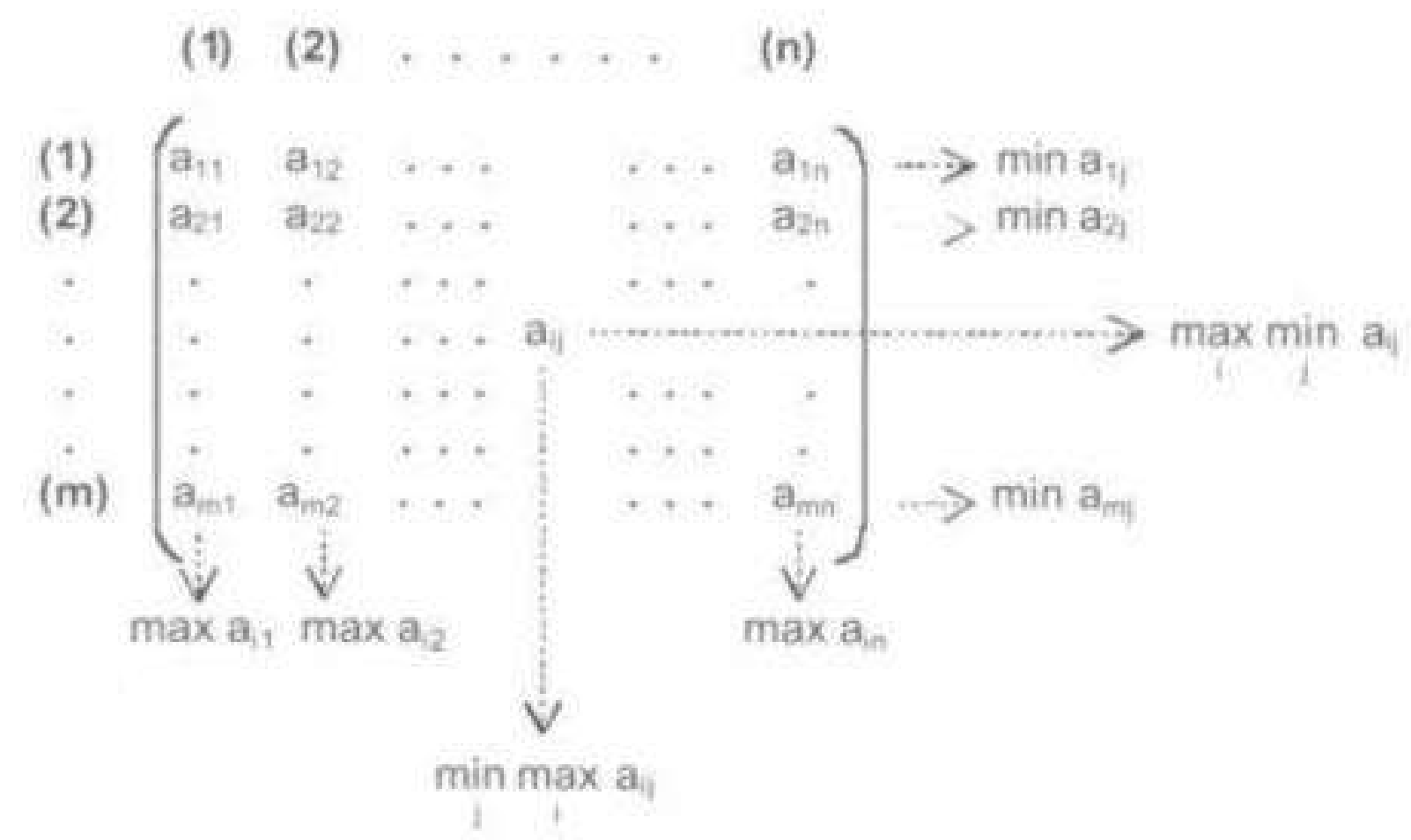

En la obra Algebra Lineal y sus Aplicaciones, cuyo autor es el suscrito, en el capitulo 7 , se exponen problemas elementales muy concretos, empleando la Teoria de Juegos, en el campo de la agricultura, en el campo de la economia, en el campo de la medicina, etc.

Finalmente, la Academia Sueca de Ciencias al otorgar el Premio NOBEL de 1994 a los tres economistas anteriormente señalados, indicó que los tres hicieron una contribución eminente a los análisis de equilibrio, la Teoria permite

realizar predicciones. La Academia dijo que la Teoria ha sido aceptada en el análisis económico y puede ser aplicada en estudios de organizaciones industriales, monopolios e incluso cuando los mercados financieros intentan determinar cómo se comportará el Banco Central. Asi, "El Equilibrio de Nash" (llamado asi por John Nash) se ha convertido en un instrumento común en todas las áreas de la Teoria Económica. 